\title{
A Study of Situation of Indigo dyed Sakon Nakhon ${ }^{1}$
}

\author{
Jiraphat Roemsri², Jantima Kheokao ${ }^{3}$ \\ Marketing Communication Program, School of Communication Arts University of \\ the Thai Chamber of Commerce
}

\begin{abstract}
A Study of Situation of Indigo dyed Sakon Nakhon. The preliminary data from in - depth interviews with representatives of manufacturers, entrepreneurs and government officials responsible for dyeing Indigo Sakon Nakhon.

The results of the study found that

Indigo dyed natural. It is a local wisdom related to knowledge, ability and experience. The ancestors learned, improved, and transcribed from generation to generation. By telling and taking action. It becomes a knowledge that has a local identity. Such as cultivation, indigo dyeing and patterns. Currently, more than 100 patterns of indigo dyed. It is a pattern that most members are skilled in weaving. The product development and application can be applied to a variety of market demand. Tatting has 2 styles 1) Ancient pattern 2) striped Applied.

The production of indigo dye is fascinating with the process of producing indigo from the plant called "Indigo" By dyeing indigo is a cool dye. The way of wisdom inherited from the ancestors. Indigo dyed cloth, thus creating a sentimental value to manufacturers and the occupant's hard to get comparable value.
\end{abstract}

Keyword: Indigo, Local, Manufacturers, Sakon Nakhon, Situation

\footnotetext{
${ }^{1}$ This paper is a part of the dissertation entitled "Brand Identity Communication Strategy of Sakon Nakhon Indigo Dyed Fabric" which received financial support from the National Research Council of Thailand.

${ }^{2}$ Doctor of Marketing Communication (candidate), School of Communication Arts, University of The Thai Chamber of Commerce

${ }^{3}$ Associate Professor Dr. Jantima Kheokao, is a Director of the Doctor of Communication Arts Program in Marketing Communication, University of the Thai Chamber of Commerce
} 


\section{Introduction}

Sakon Nakhon province has a cultural history that has a unique identity to find. And also a city with ancient historic sites that have historical value there are 6 populations of different ethnic groups, similar lifestyles and traditions. With similar lifestyles and traditions Most of them are agricultural workers. When abstaining from seasonal farming these ethnic groups are popular in the household industry: weaving or hand-woven fabrics. For household use Is a folk wisdom that has been inherited from the ancestors By means of telling, making, watching and performing by themselves There is a process of producing patterns that are inherited from prominent ancestors. Identify the identity of each ethnic group (Chintana Linphosan, 2013)

Causing the government to have a policy to push for the integration of people in the community and bringing resources in the community as well as bringing existing local wisdom to produce products Became a parish product One product To generate income And self-reliance of families, communities and between communities for products that originated from Sakon Nakhon unique identity, "Indigo dyed fabric" Is a clever local wisdom of Thai ancestors in tinting colors from natural indigo to lines that imitate more than 6,000 years Sakon Nakhon is the first province in Thailand to revive local wisdom, indigo dyed fabrics. And has developed continuously It is a charming product that is a wisdom that creates mental value for manufacturers, givers and occupiers. Difficult to compare value making the indigo dyeing fabrics valuable, also beneficial to maintaining the identity Indigo dyed fabric Sakon Nakhon (Indigo Center Sakon Nakhon Rajabhat University, 2017)

Indigo dyed fabrics are products that are related to culture, have a weakness in marketing, creating demand and recognition for customers very much, because most Indigo silk products often produce products in the same form without the development of images Modern product designs are produced in the form of their own group without knowing that consumers want the product in any form. Product image without specific identity and lack of good marketing communication (Wanna Chokbandansuk, 2015)

From the above ideas, it can be seen that marketing communication is important to the success of Sakon Nakhon Indigo dyed fabrics. Which means the number of buyers of more products As well as searching for product differences helping customers to evaluate the quality and see the difference clearly Support is important to add value to the product

Is a tool that can help find the true identity of ethnic groups Make the community proud of their own local Making it necessary to A Study of Situation of Indigo dyed fabrics Sakon Nakhon to use the information obtained to develop the appropriate marketing communication model able to truly meet the needs of consumers. 


\section{Methods and Data Collection}

My analysis is from in - depth interviews. Printed as word-by-word documents to verify the accuracy of the information by dividing the data from the interview to determine according to the study topic

Collecting data by entering the field that interviewed representatives of manufacturers, entrepreneurs and government officials who are responsible for Sakon Nakhon Indigo dyed fabrics. To conduct in - depth interviews with 20 people

In order to obtain complete information in accordance with the research objectives Where the interviewee can answer questions openly Unlimited answers To provide an opportunity for the interviewee to fully convey the most complete and true knowledge The interview with each main informant There may be more than one interview appointment since the first time may not have much information and is more familiar. When trusting, then there will be an appointment for the next interview in order to get the complete information that meets the most research objectives. By accumulating and using the interview for about 1 hour each. By selecting the main information provider using a convenient random method from the indigo dyeing cloth manufacturer group in each district who are willing to interview as a sample in this research.

\section{Results and Discussion}

The results of A Study of Situation of Indigo dyed Sakon Nakhon by collecting data with in - depth interviews from key informants found that

Indigo dyed fabric, Sakon Nakhon Is a product that uses hand - woven cotton as the main fiber in the weaving But not enough to meet the needs of consumers Causing Indigo dyed cotton cloth manufacturers And rayon cotton used The fibers are dyed with indigo natural color according to the traditional method. Make a difference from the past with only white and blue with natural colors from the bark. Is a product that has mental value Price is not the issue of people who like it. Because of the quality of the indigo dyed fabric, every manufacturer pays attention to making every piece. Like the spirit of the weaver And Sakon Nakhon indigo dyes are diverse and different in each ethnic group But have similar characteristics in the weaving and mudmee, ancient patterns Making it necessary to create the identity of indigo dyed fabrics to be outstanding By using weaving techniques And applying ancient patterns to modern or natural patterns around them, bringing mudmee to create patterns to create identity through stories or ways of life in the community. (Research and development Rajamangala University of Technology Isan Sakon Nakhon Campus, 2012) History of indigo dyeing in the northeastern region of Thailand The popular fibers used to dye natural indigo are cotton fibers, where the hand-woven cotton is the fiber that the indigo dyeing manufacturer in Sakon Nakhon has used since the past But nowadays technology is more modern Combined with customer needs Causing some manufacturers of indigo dyed fabrics to use cotton for factories Which is pure cotton that has passed the production process of the factory And another type of fiber that the manufacturer of indigo dyes in Sakon Nakhon used to be rayon cotton (Anurat Saithong and Phuwadon Sritharet, 2015) The indigo dyeing cloth 
in Lao PDR and the Socialist Republic of Vietnam Popular with hand-woven cotton lines The fibers are dyed with indigo natural color according to the traditional method. By making a difference from the past With only white and blue with natural colors from the bark (Kitikhun Hompan, 2012) In which each group has different methods of acquiring natural colors The natural colors that are currently used are from bark, core, leaves and flowers. In addition, each group also has an indigo dyeing formula with unique identities that are passed down from generation to generation.

Inheritance of indigo dyed fabric wisdom of each ethnic group has similar characteristics, most of which are characteristics of telling and act Because everything is caused by the knowledge of the successor Each group will have a way to tell. Or different methods of inheritance, consistent with (Sutisa Songleknok, 2015). Other network groups through informal demonstrations, practical exercises Making each group have a concept of creating a community learning base To draw people interested in learning In order to conserve and inherit indigo dyed cloth to remain in the community But in the present, the transfer of knowledge, production and weaving of indigo dyes has not been recorded in writing. Lacks of systematic process recording those who are skilled in weaving are mostly elderly. If those people died that knowledge may also be lost. (Siraporn Kuljittiwirach, 2013)

Marketing communication that manufacturers And the Indigo dyed fabric operator Sakon Nakhon used to communicate with consumers there are various channels. Received promotion from government and private agencies. Have printed media presented via radio, television Internet media the exhibition but most of the manufacturers and operators of Indigo dyed fabric Use online marketing communication via Facebook. And the exhibition of products by government and private agencies (Phetphrairin Upping, 2013) Linking the supplier network Large and mediumsized groups can find the market by themselves. With the exact order and participated in the exhibition of products by government and private agencies There are 2 types of small groups. The first category is assisted by the government. The second type is not the exact marketing. Participated in the trade show in the province And consignment to large groups (Indigo Center Sakon Nakhon Rajabhat University, 2017) Marketing channels or distribution channels Indigo dyeing fabric Sakon Nakhon There are products sold in the activities of the agency. Opening for tourists to visit the indigo fabric group Mobile trading online trading and manufacturers selling products through middlemen or distributors the online sales channel via Facebook Fan page is the most popular distribution channel today. Because easy access, easy to use, free of charge Causing manufacturers to not have to invest more (Jeeranan Chareonrat, 2016)

Content used to communicate to convince consumers Most of the manufacturers and operators of Indigo dyed fabrics in Sakon Nakhon Will communicate the naturalness of the product such as natural indigo Natural indigo dyed cotton Properties of indigo dyed fabric Creating a difference with modern knitting patterns or patterns Environmental care dyeing fabric production process Or indigo dyed fabric, natural color, not falling, etc. It is a communication with indigo dyeing products to consumers through natural indigo, filament, pattern and production. (Sutisa 
Songleknok, 2015) found that consumers who bought indigo dyed fabric products Will have to order or discuss exchange about indigo dyeing products such as shades design And the

characteristics of the fibers used to produce the fabric, etc., according to the views and views of the customers.

\section{Conclusion}

Based on the above study, it is seen that Sakon Nakhon province is a province in Thailand. Is a land that has a long history which is rich in arts, culture, traditions and is a cultural attraction with a variety of ethnic groups making it unique in local art and culture Which Sakon Nakhon indigo dyeing cloth is a local handicraft with distinctive patterns and identity In the past, focusing on the production process to use in daily life, emphasizing utility rather than beauty, having a simple product model, product characteristics of each district will be different

But at present, when the indigo dyeing is revived Causing the indigo dyeing cloth to become an important cultural product of Sakon Nakhon Province The wisdom of indigo dyed fabrics is conveyed through stories and stories. Than becoming an indigo dyed fabric until being determined as an important strategy of Sakon Nakhon Province Along with being driven to make indigo dyed fabrics widely accepted in the context of globalization

Indigo dyed fabric, Sakon Nakhon, in general, mostly blue fabric Use mudmee technique to create patterns Indigo dyeing Sakon Nakhon of each ethnic group in many countries still maintains a unique style. Indigo dyed fabrics, in addition to science and art Also means the way of life, beliefs, desserts, customs and rituals Inherited from the past But with the development of things according to the age, both the pattern of dyeing and pattern

Indigo dyed fabric, Sakon Nakhon when it was made into a product Consumer groups have changed from the original dyeing with indigo, it changed into a tie with other types of wood to find the novelty of the fabric. Until causing the indigo to reduce its role this change of process is caused by the indigo dyeing cloth manufacturers getting more ideas from the outside world. Have seen many processes that will increase the value of the product Therefore thinking of inventing new inventions to meet the needs of consumers Causing the distraction of the indigo to the dyeing of new materials But still use the name of indigo dyed fabric as a selling point Which may cause the indigo dyeing fabric to lose its identity shortly

The reintroduction of the indigo blue fabric has resulted in more indigo fabrics. Indigo cloth users are no longer villagers. But being a middle class group with salaries and purchasing power of indigo because each indigo fabric has a relatively high price the market mechanism makes it a valuable and expensive cultural product. Different from traditional indigo dyed fabrics, which are cloths of villagers used to wear in farming. Rice farming and daily life The group of people who wear indigo dyed fabrics is therefore a group of unique tastes. In addition, the state or government organizations are involved in the management of indigo dyed fabrics. Supporting, encouraging and pushing the group to develop the ability to produce indigo fabrics and designs to 
make indigo fabrics accessible to a wider consumer market causing an increase in the number of Indigo dyed fabric manufacturers in Sakon Nakhon

Making indigo dyed fabric which is wisdom to become a cultural product that can be sold. Not caused by villagers but being a part to promote and support from the government In addition, government agencies are encouraged to wear indigo dyed clothes every Friday. Making indigo cloth in the perception and familiarity of the common people finally became the identity of Sakon Nakhon Province which such factors help promote the indigo dyeing cloth to be more widely known. It can be concluded that Sakon Nakhon indigo dyed fabrics use fibers from natural cotton, cotton, factories and rayon cotton, dyed with natural indigo water Make a difference by using natural colors from the bark. In addition, the use of mudmee techniques to create various patterns including ancient patterns. And applied patterns the weaving technique depends on the experience of the weavers. The production of indigo dye is fascinating with the process of producing indigo from the plant called "Indigo" By dyeing indigo is a cool dye. The way of wisdom inherited from the ancestors. Indigo dyed cloth, thus creating a sentimental value to manufacturers and the occupant's hard to get comparable value. 


\section{References}

\section{Book}

Chintana Linphosan. (2013). A Value of local a Stand culture identity and adaptation to make local product for adding cost in and cultural tourism: Case study of local weaving group in Sakon Nakhon province. Sakon Nakhon: Sakon Nakhon Rajabhat University. Indigo Center Sakon Nakhon Rajabhat University. (2017). Indigo king of natural dyes. Sakon Nakhon: Sakon Nakhon Rajabhat University.

Phetphrairin Upping. (2013). Group Management Process and Community Enterprise Development: A Case study of Sakon Nakhon Province. Faculty of Industry and Technology Rajamangala University of Technology Isan Sakonnakhon Campus. Jeeranan Chareonrat. (2016). Social media Marketing Channel of Dokkaew Stripe, Si Kao Blanket: A case study in Weavers Groups in Tao Ngoi District, Sakon Nakhon Province, Thailand. Faculty of Management Science Sakon Nakhon Rajabhat University. Siraporn Kuljittiwirach. (2013). Knowledge Management of Indigo Dye Cloth Weaving in Ban Non Sa - at, Na Ngua Sub - District, Nawa District, Nakhon Phanom Province. Master of Agriculture Sukhothai Thammathirat Open University.

\section{Journal}

Sutisa Songleknok. (2015). Knowledge Management Process of Indigo Dyed Cloth Community Business. KKU Res J (GS). Volume 15, Issue 3. pp. 1 - 14.

Wanna Chokbandansuk. (2015). Competitive Strategy Development Based on Creative Economy for Tai - Yuan Locally Woven Cloth in Ratchaburi Province. SDU Res. J. Volume 11, Issue 1. pp. 17 - 32. 